\title{
Structural adaptive anisotropic recursive filter for blind medical image deconvolution
}

Tanawat Kwanpak, Paramate Horkaew

School of Computer Engineering, Suranaree University of Technology, Thailand

\begin{tabular}{|c|c|}
\hline Article Info & ABSTRACT \\
\hline Article historys: & Performance of radiographic diagnosis and therapeutic intervention heavily \\
\hline Received Nov 29, 2018 & $\begin{array}{l}\text { depends on the quality of acquired images. Over decades, a range of pre- } \\
\text { processing for image enhancement has been explored. Among the most recent }\end{array}$ \\
\hline Revised May 1, 2019 & proposals is iterative blinded image deconvolution, which aims to identify the \\
\hline Accepted May 15, 2019 & $\begin{array}{l}\text { inheritant point spread function, degrading images during acquisition. Thus } \\
\text { far, the technique has been known for its poor convergence and stability and }\end{array}$ \\
\hline Keywords: & $\begin{array}{l}\text { was recently superseded by non-negativity and support constraints recursive } \\
\text { image filtering. However, the latter requires a priori on intrinsic properties of }\end{array}$ \\
\hline Anisotropic & imaging sensor, e.g., distribution, noise floor and field of view. Most \\
\hline Deconvolution & $\begin{array}{l}\text { importantly, since homogeneity assumption was implied by deconvolution, } \\
\text { recovered degrading function was global disregarding fidelity of underlying }\end{array}$ \\
\hline NAS-RIF & $\begin{array}{l}\text { objects. This paper proposes a modified recursive filtering with similar non- } \\
\text { negativity constraints, but also taking into account local anisotropic structure } \\
\text { of content. The experiment reported herein demonstrates its superior } \\
\text { convergence property, while also preserving crucial image feature. }\end{array}$ \\
\hline
\end{tabular}

Copyright (C) 2019 Institute of Advanced Engineering and Science. All rights reserved.

\section{Corresponding Author:}

Paramate Horkaew, School of Computer Engineering, Suranaree University of Technology, 111 University Avenue, Nakhon-Rachasima, Thailand. Email: phorkaew@sut.ac.th

\section{INTRODUCTION}

Recent advances in medical imaging technology has so far enabled high performance computerized radiographic diagnosis and therapeutic intervention [1-5]. More specifically, it has been widely applied, for examples, in patient specific anatomical modeling, lesion extraction and more recently in unsupervised deep learning [6]. Thus far, degradation is one of major impeding factors in their success. Although in practice, it is led by a series of complex processes imaging signal underwent during acquisition, for simplicity, the term is typically characterized by linear deconvolution of blurring kernel and an additive noise [7], as expressed in (1).

$$
\mathrm{g}(x, y)=f(x, y) * h(x, y)+n(x, y)
$$

where $g$ and $f$ are degraded and (presumably) original images, respectively. In the spatial domain of (x, y) $\in$ $\mathbf{R}^{2}, h$ and $n$ are convolutional kernel and noise, respectively. In analyzing degradation process, $h$ is sometimes referred to as blur filter or, in our context, point spread function (PSF). Reconstructing the original image $f$, given the degraded $g$ and a priori on (or sometimes, unknown) noise model, $n$, is however not trivial. Its key element involves estimating a PSF and its respective inverse $\left(h^{-1}\right)$. This process is called deconvolution and depicted in Figure 1. 


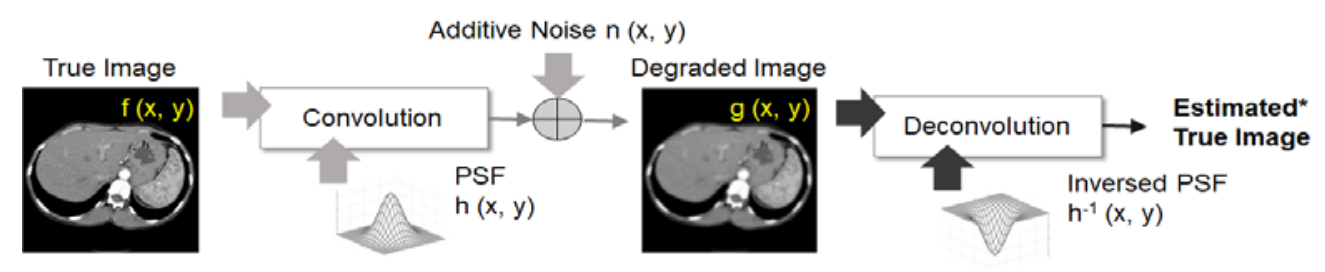

Figure 1. Direct filtering model of convolution and deconvolution

Depending on assumption of the degrading model, PSF can be estimated by calculating its governing (for example, blurring) parameters [8, 9]. However, several other factors, such as out of focus, motion, and geometrical distortion, etc. may have equally contributed to degraded image quality. Identifying these simple model parameters was unable to completely restore such adverse effects. Determining types of degradation, their ranking and interactions, indeed are not trivial, especially without access to imaging modality calibration. Another approach, called blind image deconvolution (BID) [7, 10, 11], tackled this problem by estimating each element in PSF kernel/ matrix (or its inverse), subject to some criteria, but without prior on degradation sources. BID combines PSF estimation and deconvolution into a single reciprocal process. Estimation of PSF is usually done by iteratively updating vectorized kernel using a gradient descent variant [12], whereby in each cycle its values are varied with respect to pre-defined objective function and constraints. Provided that an optimum exists, upon convergence, resultant inversed PSF are able to closely recover the true non-degraded image. It can be noted that noise model was not incorporated into the inversed PSF and hence would have caused instability in case of low SNR. Other studies thus opted for operations in frequency domain, in which linear property of convolution can be exploited $[13,14]$, i.e., $\mathbf{F}^{*}(\mathrm{u}, \mathrm{v})=\mathbf{G}(\mathrm{u}, \mathrm{v}) \mathbf{H}^{-1}(\mathrm{u}, \mathrm{v})$, where capital letters refer to (estimated) true, degraded images and PSF in frequency domain, respectively. Accordingly, noise may be dismissed by selectively processing only in the lower frequency spectrum. Although iterative variants exist, PSF estimation in frequency domain are of close-form and more efficient, compared to that on spatial one. However, its main drawback was that knowledges about noise properties are prerequisite, without which severe instability could occur. To remedy this adverse effect, Wiener filter [15, 16], Wavelet [17] and Curvelet [18] based methods were proposed.

Note may be drawn from the literature that both spatial and frequency domain operations have their pros and cons. While the former can greatly benefit from straightforward yet intuitive constraints imposition, the latter is more efficient, with available fast spatial-frequency conversion algorithms. BID on both domains, called a nonnegativity and support constraints recursive filtering (NAS-RIF) [7, 19] was introduced and recently enhanced [20-26]. Its primary contribution was to overcome instability issue found in conventional BID. As its name suggested, NAS-RIF imposes irreducible, absolutely summable, i.e., $(\Sigma\|\mathbf{h}\|<\infty)$, and being invertible, i.e., $\mathbf{h}^{-1}$, properties on a PSF, while maintaining the same assumption (i.e., real value and positive definite) on the true image as IBD. The support on the image was defined within a region of interest (ROI). This has made NAS-RIF particularly suitable for medical imaging, where anatomical object is generally acquired in the center of a matrix and surrounded by uniform background. Specifically, NAS-RIF divided the degraded image into two regions, i.e., inside and outside support $\left(\mathrm{D}_{\text {sup }}\right)$, whose cost functions were determined with different objectives and constraints. A generic NAS-RIF algorithm is summarized in Figure 2.

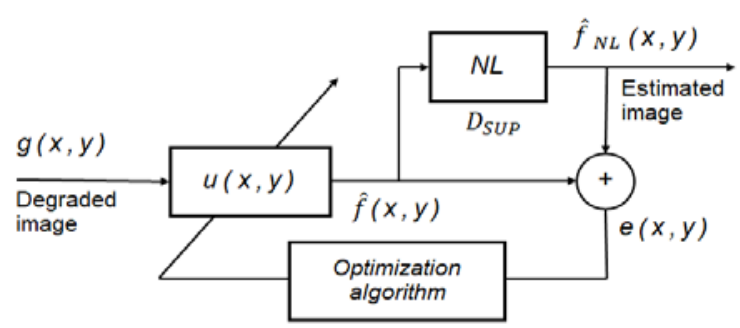

(a)

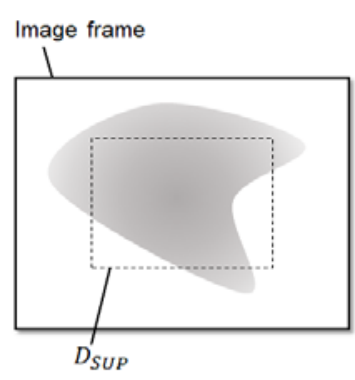

(b)

Figure 2. (a) Generic NAS-RIF algorithm [7] and (b) definition of support region ( $\left.\mathrm{D}_{\text {sup }}\right)$ 
Given a degraded image $g(\mathrm{x}, \mathrm{y})$, NAS-RIF recursively determines the optimal inversed PSF, denoted here as $\mathrm{u}(\mathrm{x}, \mathrm{y})$. At each iteration the elements of $\mathrm{u}$ are optimally adjusted subject to a cost function, consisting of negativity penalizes for pixels within and outside support region. Some enhanced proposal suggested additional regularization constraints, e.g., DC gain [21] or more realistic boundary support [22]. Noise issues were elevated by using designated filters [23-25]. For better performance nonetheless, deconvolution was performed in the frequency domain.

a. Definition

The summary of NAS-RIF algorithm (see text for detailed explanation):

- $\left.\hat{f}_{k}\right) x, y\left(\quad\right.$ : Estimate of the true image at $\mathrm{k}^{\text {th }}$ iteration

- $\left.\underline{u}_{k}\right) x, y\left(\quad\right.$ : FIR filter parameters of dimension $N_{x u} \times N_{y u}$ at iteration $\mathrm{k}$

- J( $\left.\underline{u}_{k}\right) \quad$ : Cost function, given parameter setting $u_{k}$

- $\nabla J\left(u_{k}\right) \quad:$ Gradient of $J$ at $u_{k}$

b. Set initial condition $) k=0($

- Set FIR filter $u_{k}(x, y)$ to all zeros

c. At each iteration $) k(: k=0,1,2, \ldots$

- $\hat{f}_{k}(x, y)=u_{k}(x, y) * g(x, y)$

- $\hat{f}_{N L}(x, y)=N L\left[\hat{f}_{k}(x, y)\right]$

- Minimize routine to update FIR filter parameters )conjugate gradient routine(.

$$
\begin{aligned}
& {\left[\Delta J\left(\underline{u}_{k}\right)\right]^{T}=\left[\frac{\partial J\left(u_{k}\right)}{\partial u(1,1)} \frac{\partial J\left(u_{k}\right)}{\partial u(1,2)} \cdots \frac{\partial J\left(u_{k}\right)}{\partial u\left(N_{x u}, N_{y u}\right)}\right]} \\
& \text { where } \frac{\partial J\left(\underline{u}_{k}\right)}{\partial u(i, j)}=2 \sum_{(x, y) \in D_{s u p}} \hat{f}^{2}(x, y)\left[\frac{1-s g n(\hat{f}(x, y))}{2}\right] g(x-i+1, y-j+1) \\
& +2 \sum_{(x, y) \in \bar{D}_{\text {sup }}}\left[\hat{f}_{k}(x, y)-L_{B}\right] g(x-i+1, y-j+1) \\
& +2 \gamma\left[\sum_{\forall(x, y)} u_{k}(x, y)-1\right] \\
& \beta=\left(<\nabla J\left(\underline{u}_{k}\right)-\nabla J\left(\underline{u}_{k-1}\right), \nabla J\left(\underline{u}_{k}\right)>\right) /\left(<\nabla J\left(\underline{u}_{k-1}\right), J\left(\underline{u}_{k-1}\right)>\right) \\
& \text { If } k=0, d_{k}=-\nabla J\left(\underline{u}_{k}\right)
\end{aligned}
$$

otherwise $d_{k}=-\nabla J\left(\underline{u}_{k}\right)+\beta d_{k-1}$

$$
\underline{u}_{k-1}=\underline{u}_{k}+t d_{k}
$$

- Increment $k$

- Repeat step 3) until convergence.

Despite its great stability, shortcoming of NAS-RIF was slow convergence rate. Moreover, the inversed PSF $(u)$ was a compromise between two penalize terms, derived from pixel intensity. This paper, therefore proposes a structural adaptive anisotropic term being introduced in iterative optimization. It was computed, taking into account local orientation pattern of object structure. Its main contribution was not only emphasizing on updates in favor of feature preservation, but also promoting faster convergence as fidelity was enhanced. The remaining of this paper is organized as follow: Section 2 describes the proposed method in more detail. Section 3 reports experimental results and relevant analyses. Finally, section 4 states concluding remark of this study.

\section{RESEARCH METHOD}

This paper partly adopted conventional NAS-RIF following the process, depicted in Figure $2 \mathrm{a}$. It iteratively adjusted FIR filter, $u$, and simultaneously its output, that was an intermediate estimation of the true image, $f^{*}$. To ensure nonnegativity and support constraints, this image was projected onto a non-linear (NL) space that diminished pixel intensities outside the support region $\left(D_{\text {sup }}\right)$ to that of the background $\left(L_{B}\right)$. The corresponding non-expansive map, $\mathrm{f}_{\mathrm{NL}}$ were then subtracted with its precedent, resulting in error matrix, given in (2) [20].

Structural adaptive anisotropic recursive filter for blind medical image ... (Tanawat Kwanpak) 


$$
e(x, y)=\sum_{\forall(x, y)}\left[\hat{f}_{N L}(x, y)-\hat{f}(x, y)\right]^{2}
$$

where

$$
\hat{f}_{N L}(x, y)= \begin{cases}\hat{f}(x, y) & \text { if } \hat{f}(x, y) \geq 0 \text { and }(x, y) \in D_{S U P} \\ 0 & \text { if } \hat{f}(x, y)<0 \text { and }(x, y) \in D_{S U P} \\ L_{B} & \text { if }(x, y) \in \bar{D}_{S U P}\end{cases}
$$

Instead of simply minimizing this matrix, which would prematurely bring estimation to halt, the error image was then divided by supporting region. Ideally, object pixels bled outside $\mathrm{D}_{\text {sup }}$ due to degrading (e.g., blurring) PSF should be drawn back inside, leaving only the background. This could be achieved by an edgeenhancing FIR. However, exaggerating this adjustment could lead to negative pixels by deconvolution. To maintain the balance of these constraints, basic NAS-RIF defined the cost function to penalize negative pixels inside the $\mathrm{D}_{\text {sup }}$ and background discrepancies outside. According to the recent modification [21], irreducibility of FIR was also ensured by regularization. The cost function adopted in this study was thus given in (3). Using this function, it is trivial to prove that its gradient with respect to an FIR element. Given the cost function J and its respective gradient, $\nabla \mathrm{J}$, iterative non-linear optimization was done using a conjugate gradient method [26].

$$
J=\sum_{(x, y) \in D_{S U P}} \hat{f}^{2}(x, y)\left[\frac{1-\operatorname{sgn}(\hat{f}(x, y))}{2}\right]+\sum_{(x, y) \in \bar{D}_{S U P}}\left[\hat{f}(x, y)-L_{B}\right]^{2}+\gamma\left[\sum_{\forall(x, y)} u(x, y)-1\right]^{2}
$$

where sgn and $\gamma$ were, respectively, a signed function and an empirical factor weighting FIR regularization. Upon convergence, when the difference between successive true image estimations fell within a pre-defined threshold, the resultant FIR filter was then applied to the degraded image, g, producing the final true image restoration.

It was, however, reported in the recent NAS-RIF literature that noise reduction and a priori on underlying pixel distributions are essential determinant in its stability and restoration result. In addition, penalizing cost function, while sufficient for typical photographic images, did not consider structural fidelity in an image, hence undermining anatomical features, crucial for the subsequent analyses. Inspired by intuitive constraints augmentation found in the recent works, this paper therefore incorporated an anisotropic measure into NAS-RIF optimization. Structural anisotropic measure was introduced in [27] and later improved in [28]. In those studies, it was used to orient and adjust the extent of an adaptive FIR filter so that it aligned with underlying pixel orientation pattern. Anisotropic measure within neighborhood surrounding a pixel, p, is given in (4).

$$
\operatorname{ani}(p)=\frac{\left\{\iint\left(\frac{\partial f}{\partial x}\right)^{2}-\left(\frac{\partial f}{\partial y}\right)^{2} d x d y\right\}^{2}+\left\{\iint 2\left(\frac{\partial f}{\partial x}\right)\left(\frac{\partial f}{\partial y}\right) d x d y\right\}^{2}}{\left\{\iint\left(\frac{\partial f}{\partial x}\right)^{2}+\left(\frac{\partial f}{\partial y}\right)^{2} d x d y\right\}^{2}}
$$

Figure 3 depicts a sample image with three selected neighborhood centers with different local orientation patterns and their respective anisotropic values.

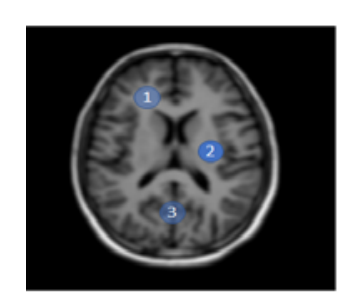

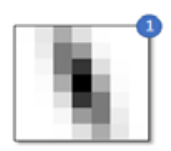

ani $=0.70$

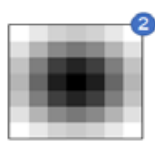

ani $=0.10$

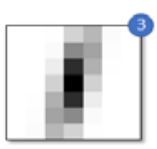

ani $=0.75$

(1) Right orientation pattern

(2) Isotropic pattern (low g)

(3) Strong left orientation pattern

Figure 3. A sample image with three selected centers and corresponding anisotropic values

In order to avoid further complicating the cost function (3), which would inevitably cause even more local minima, this study instead encouraged feature preservation by adjusting updating step size $t$ according to relative anisotropic strength, as expressed in (5). 


$$
t=\alpha\left(\frac{\sum_{\forall(x, y)} \operatorname{ani}\left(\hat{f}_{k}(x, y)\right)}{\sum_{\forall(x, y)} \operatorname{ani}\left(\hat{f}_{k-1}(x, y)\right)}\right)^{2}
$$

where $\alpha$ was a typical stepping size taken in each iteration.

The benefits of adjusting step size according to total relative anisotropic were two folds. Firstly, while steering away from non-negativity, original NAS-RIF cost function tended to stumble around an overly smoothing kernel. With anisotropic controlled step size, on the other hand, as the bled pixels were gathered inside, implying more pronounced object boundaries, the relative anisotropic measure also increased and so was the confidence in such adjustment. This effectively accelerated NAS-RIF convergence. Secondly, involving anisotropic measure into the optimization also helped lessen the dependency on having to meticulously initialize the supporting region [22]. It is also worth emphasizing here that, anisotropic measure was computed within a neighborhood of specified extent and not from an isolated pixel. It was thus robust against imaging noise [27].

\section{RESULTS AND ANALYSIS}

Without loss of generalization, the proposed enhanced NAS-RIF algorithm was examined by applying to both synthetic and medical images corrupted with known degradation. The images were encoded as 2D matrix of grayscale intensities, whose values were stored and processed in floating point format.

\subsection{Anisotropic strength as image contrast regularization}

As pointed out in [20] and subsequent works, trivial all-zero condition could be prevented by imposing a total sum constraint on FIR kernel. We found that it did not, however, rectify a uniform FIR kernel that would bring the image contrast tremendously down to an all-grey. To demonstrate that in addition to structural pattern [27] anisotropic measure is also responsive to such condition (and thus was a viable means of circumvent this problem) relationships between contrast appearances and respective total anisotropic strength are shown in Figure 4.

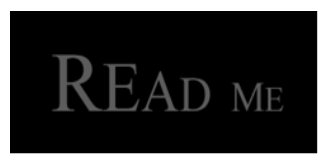

ani $=3.869$

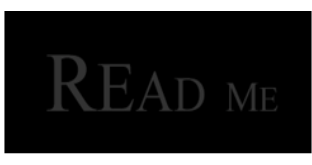

ani $=0.260$

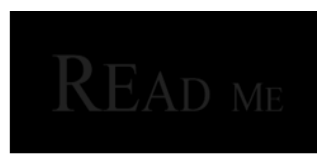

ani $=0.044$

Figure 4. Relationships between synthetic image appearances and their anisotropic measures

During an early stage of optimization, image contrast could be regularized by anisotropic strength. More specifically, as the FIR proceeded away from trivial all-zeros, the measure helped increasing its confidence by further stepping in that direction.

\subsection{Visual enhancement}

An MR scan of a human brain on a uniform background whose matrix size was 350x350 pixels, was then employed in the next experiment. Comparison between enhancement made by a generic NAS-RIF and the proposed enhancement against an original MR image are illustrated in Figure 5. The results are snapshots at the $80^{\text {th }}$ iteration.

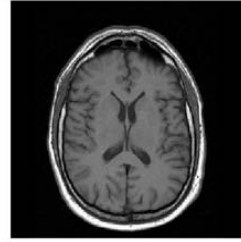

Original

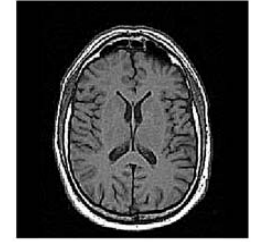

Generic NAS-RIF

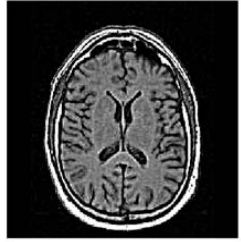

Proposed NAS-RIF

Figure 5. Comparison between a generical (middle) and proposed NAS-RIF (right) enhancement

Structural adaptive anisotropic recursive filter for blind medical image ... (Tanawat Kwanpak) 
The resultant inversed FIR, $\mathrm{u}$, brought sharper edge and better separation between tissue and the skull. Dynamic range of pixel intensities was much improved, compared to the generic NAS-RIF. It is also worth noted here that, instead of thousands of iterations usually required by a generic NAS-RIF to converge [7, 19], the proposed enhanced NAS-RIF gave an estimation with already higher fidelity and contrast at much early cycles. Moreover, no other priors were needed.

\subsection{Relationship between anisotropic strength and visual enhancement}

Figure 6 compares anisotropic strengths during the first $80^{\text {th }}$ iterations between a generic and the proposed NAS-RIF implementation, and respective enlarged original image estimations. It is evident from the graph that in the proposed implementation, the strength accelerated at faster rate, which well corresponded to much enhanced appearance. It was thus a suitable metric for a NAS-RIF optimization constraint and well conformed to the preliminary hypothesis of this study.

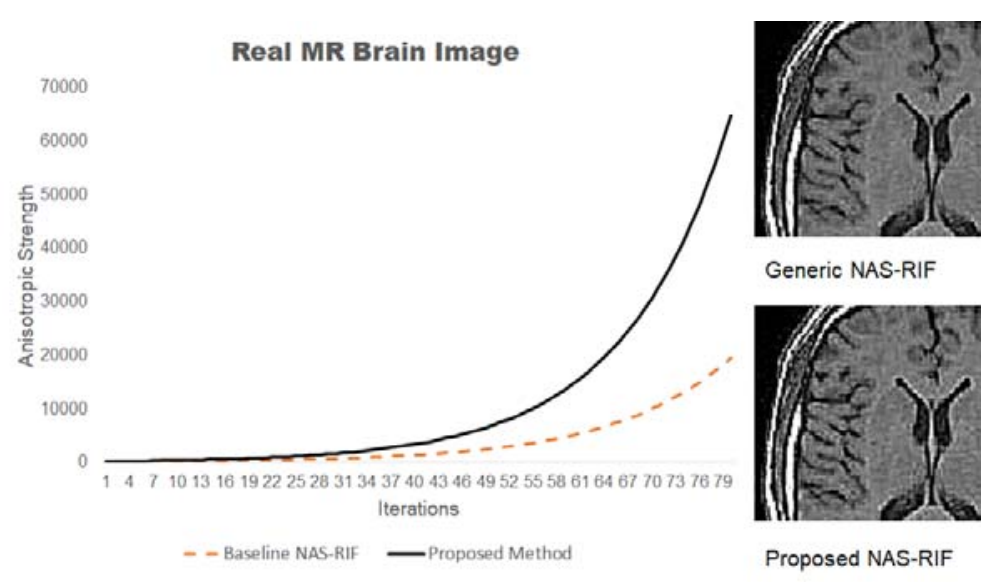

Figure 6. Comparison of anisotropic strengths (left) between generic (top-right) and proposed (bottom-right) NAS-RIF implementations

\subsection{Numerical assessments}

To quantitatively elucidate the proposed NAS-RIF scheme, especially in terms of noise immunity, numerical assessment was performed on simulated adulteration To this end, a phantom image was degraded with Gaussian blur and polluted with Rician noise (to emulate what happens in MR acquisition). Peak SNR $[29,30]$, was then computed for the original, adulterated, and generic and proposed NAS-RIF enhanced images. The peak SNR (PSNR) and corresponding restored images at $40^{\text {th }}$ iteration were listed in Table 1 and shown in Figure 7, respectively.

Table 1. PSNR of a brain phantom image, after enhanced with generic and proposed NAS-RIF methods

\begin{tabular}{cc}
\hline Enhanced Method & PSNR (dB) \\
\hline Generic & 25.392 \\
Proposed & 25.449 \\
\hline
\end{tabular}

$$
\begin{aligned}
& P S N R=10 \log \frac{P e a k^{2}}{M S E} \\
& M S E=\frac{1}{W \times H} \sum|f-g|^{2}
\end{aligned}
$$

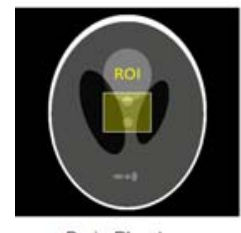

Brain Phantom

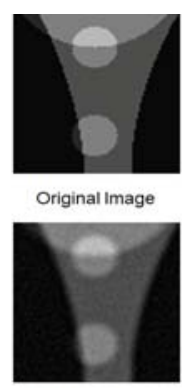

Generic NAS-RIF

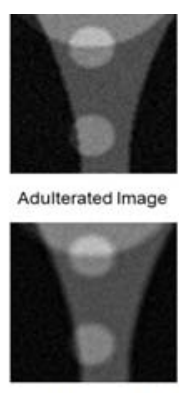

Proposed NAS-RIF

Figure 7. Visual comparisons of original, adulterated and generic and proposed NAS-RIF enhanced images 
Although quantitatively and visually, there was only slight improvement in PSNR over the generic NAS-RIF, the proposed method was equally if not better immune to additive noise. To elucidate that the proposed enhanced NAS-RIF could equally well applied to other images, the anisotropic strength and convergence were compared against the baseline one. The experiments were performed on README, Brain Phantom and Real Abdominal CT images. It is evident from the Figure 8 that the proposed method consistently outperformed the baseline one in all examples.
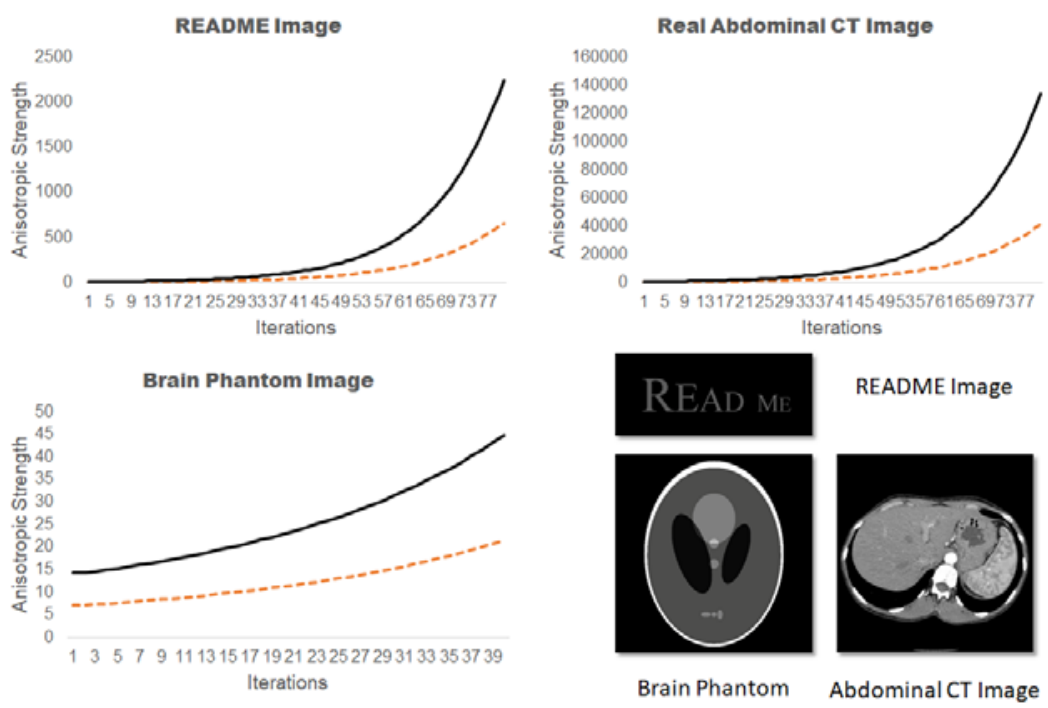

Figure 8. Comparison of anisotropic strengths of README (top-left), brain phantom (botom-left) and real abdominal CT (top-right) images between the proposed (solid-line) and baseline (dash-line) NAS-RIF implementations and pictorial annotation of each experiment is shown in bottom right

\section{CONCLUSION}

Blind image deconvolution is an ill-posed problem that was designed to restore the true image, undergone degradation by an unknown PSF and possibly by random noise. Variational BID elevates this problem by iteratively estimate the PSF (or its inversed) subject to some pre-defined criteria. NAS-RIF is another well accepted variation BID that imposed non-negativity and supports constraints over a sequence of restored image, during the optimization. Nonetheless, it is prone to noise and had low convergence rate. Many attempts had been made in the literature to address these issues, by suggesting various FIR regularization schemes, selectively filtering the projected image, or accurately defining the object support, etc.

This paper put emphasis on quality of the shape and object definition and thus proposing a structural adaptive metric, i.e., anisotropic strength. Its advantages are robustness against noise and intuitively representing characteristics of local orientation pattern. Unlike other recent works, this paper did not augment anisotropic strength into an already complicate NAS-RIF cost function, or else it would have caused minima traps and created another unnecessary expression to be weighted and balanced. On the contrary, it was used simply to adjust the step size in each kernel update. The benefits were two folds; it accelerated convergence as object boundaries became more pronounced and structural appearance. It concisely represented the structural appearance of underlying object and thus lessen the need of precise initial support.

The experimental results reported herein confirmed visually and numerically that the proposed NASRIF had much higher convergence rate, offered restoration of better quality, and was equally immune to synthetic noise. It was therefore believed that the proposed method could offer a new direction toward improving the performance of the widely adopted NAS-RIF, especially in the fields of medical imaging, computer aided diagnosis (CAD), and digital anatomy.

\section{ACKNOWLEDGEMENT}

This work was supported by Suranaree University of Technology, under One Research One Graduate (OROG) grant scheme. 


\section{REFERENCES}

[1] Lee, S., Horkaew, P., Darzi, A. and Yang, G., "Statistical shape modelling of the levator ani with thickness variation," Lecture Notes in Computer Science, vol. 3216, pp. 258-265, 2004.

[2] Hasan, A., "A hybrid approach of using particle swarm optimization and volumetric active contour without edge for segmenting brain tumors in MRI scan," Indonesian Journal of Electrical Engineering and Informatics (IJEEI), vol. 6(3), pp. 292-300, 2018.

[3] Suksuphew, S. and Horkaew, P., "Hyperplanar morphological clustering of a hippocampus by using volumetric computerized tomography in early Alzheimer's disease," Brain Sciences, vol. 7(11), pp. 155, 2017.

[4] Singh, K., Kumar, S. and Kaur, P., "Automatic detection of rust disease of Lentil by machine learning system using microscopic images," International Journal of Electrical and Computer Engineering (IJECE), vol. 9(1), pp. 660-666, 2019.

[5] Sigit, R., Roji C.A., Harsono, T. and Kuswadi, S., "Improved echocardiography segmentation using active shape model and optical flow,” TELKOMNIKA, vol. 17(2), pp. 809-818, 2019.

[6] Litjens, G., et al., "A survey on deep learning in medical image analysis," Medical Image Analysis, vol. 42, pp. 60-88, 2017.

[7] Kundur, D. and Hatzinakos, D, "A novel blind deconvolution scheme for image restoration using recursive filtering," IEEE Transactions on Signal Processing; vol. 46(2), pp. 375-390, 1998.

[8] Yitzhaky, Y. and Kopeika, N. S., "Identification of blur parameters from motion blurred images," Graphical Models and Image Processing, vol. 59(5), pp. 310-320, 1997.

[9] Flusser, J., Suk, T. and Saic, S., "Recognition of blurred images by the method of moments," IEEE Transactions on Image Processing, vol. 5(3), pp. 533-538, 1996.

[10] Kundur, D. and Hatzinakos, D., "Blind image deconvolution," IEEE Signal Processing Magazine, vol. 13(3), pp. 43-64, 1996.

[11] Kundur, D. and Hatzinakos, D., "Blind image deconvolution revisited," IEEE Signal Processing Magazine, vol. 13(6), pp. 61-63, 1996.

[12] Weickert, Joachim, “Anisotropic diffusion in image processing," Stuttgart: Teubner, vol. 1, 1998.

[13] Cannell, M. B., McMorland, A. and Soeller, C., "Image enhancement by deconvolution," Handbook of biological confocal microscopy, pp. 488-500, 2006.

[14] Xu, L., Tao, X. and Jia, J., "Inverse kernels for fast spatial deconvolution," European Conference on Computer Vision, Springer, Cham, pp. 33-48, 2014.

[15] Ramadan, Z. M., "Effect of kernel size on Wiener and Gaussian image filtering," TELKOMNIKA, vol. 17(3), pp. 1455-1460, 2019.

[16] Javier, P. et al., "Adaptive Wiener denoising using a Gaussian scale mixture model in the wavelet domain," in Proc. of International Conference on Image Processing. IEEE, vol. 2, 2001.

[17] Hamiane, M. and Saeed, F., "SVM Classification of MRI Brain Images for Computer-Assisted Diagnosis," International Journal of Electrical and Computer Engineering (IJECE), vol. 7(5), pp. 2555-2564, 2017.

[18] Starck, J., Candès, E.J. and Donoho, D. L., "The curvelet transform for image denoising," IEEE Transactions on image processing, vol. 11(6), pp. 670-684, 2002.

[19] Kundur, D. and Hatzinakos, D., "A novel recursive filtering method for blind image restoration," Proc. IASTED International Conference on Signal and Image Processing, pp. 428-431, 1995.

[20] Kundur, D., Hatzinzkos D., "On the global asymptotic stability of the NAS-RIF algorithm," Proc. International Conference on Image Processing, pp. 73-76, 1996.

[21] Ong, C. A. and Chambers J. A., "An enhanced NAS-RIF algorithm for blind image deconvolution," IEEE Transactions on Image Processing, vol. 8(7), pp. 988-992, 1999.

[22] Maysuyama, M., Tanji, Y. and Tanaka, M., "Enhancing the ability of NAS-RIF algorithm for blind image deconvolution," Proc. IEEE International Symposium on Circuits and Systems, pp. 553-556, 2000.

[23] Wang, H., Qi, S. and Liu, Y., "An improved NAS-RIF algorithm based on the lifting scheme for blind image restoration," IEEE Circuits, Communications and Systems, pp. 423-426, 2009.

[24] Yang, Y., Yang, Z., Shen, T. and Chen, B., "ENAS-RIF algorithm for image restoration," SPIE Photonics Asia, vol. 1: 85581Z-85581Z-8, 2012.

[25] Siddhichai, S. and Chambers, J. A., "Wavelet transform-based noise reduction schemes to improve the noise sensitivity of the NAS-RIF algorithm for blind image deconvolution," Proc. IEEE Signal Processing Conference, vol. 4, 2015

[26] Raid, A. M., et al., "Fast NAS-RIF algorithm using iterative conjugate gradient method," Signal and Image Processing, vol. 5(2), p. 63, 2014.

[27] Yang, G. Z., Burger, P., Firmin, D. N. and Underwood, S. R., "Structure adaptive anisotropic image filtering," Image and Vision Computing, vol. 14, pp. 135-145, 1996.

[28] Wu, J., Feng, Z. and Ren, Z., "Improved structure-adaptive anisotropic filter based on a nonlinear structure tensor," Cybernetic and Information Technology, vol. 14(1), pp. 112-127, 2014.

[29] Sai, K. A. and Ravi, K., "An efficient filtering technique for denoising colour images," International Journal of Electrical and Computer Engineering (IJECE), vol. 8(5), pp. 3604-3608, 2019.

[30] Yalman, Y. and Erturk, I., "A new color image quality measure based on YUV transformation and PSNR for human vision system,” Turkish Journal of Electrical Engineering \& Computer Sciences, vol. 21(2), pp. 603-612, 2013. 